\title{
Nonnegative solutions of the heat equation on rotationally symmetric Riemannian manifolds and semismall perturbations
}

Minoru Murata

\begin{abstract}
Let $M$ be a rotationally symmetric Riemannian manifold, and $\Delta$ be the Laplace-Beltrami operator on $M$. We establish a necessary and sufficient condition for the constant function 1 to be a semismall perturbation of $-\Delta+1$ on $M$, and give optimal sufficient conditions for uniqueness of nonnegative solutions of the Cauchy problem to the heat equation. As an application, we determine the structure of all nonnegative solutions to the heat equation on $M \times(0, T)$.
\end{abstract}

\section{Introduction}

We consider nonnegative solutions of the heat equation

$$
\partial_{t} u=\Delta u \text { in } M \times(0, T),
$$

where $0<T \leq \infty$ and $M$ is a rotationally symmetric Riemannian manifold. The structure of all nonnegative solutions of (1.1) is well understood in the following two cases [SSP] and [UP] (for precise statements, see Fact AT and Theorem 1.4 below):

[SSP] (semismall perturbation) The constant function 1 is a semismall perturbation of $-\Delta+1$ on $M$ (see [11]).

2000 Mathematics Subject Classification: 35K05, 58J99, 35C15, 35B20, 31C35, 31C12, 35J99, 35K15.

Keywords: Heat equation, rotationally symmetric Riemannian manifold, nonnegative solution, integral representation, uniqueness, semismall perturbation, Laplace operator, Martin boundary. 
[UP] (uniqueness for the positive Cauchy problem) Any nonnegative solution of the Cauchy problem to the heat equation is determined uniquely by the initial data (see [1]).

The purpose of this paper is to establish a necessary and sufficient condition for [SSP], and to give optimal sufficient conditions for [UP].

Now, in order to state our main results, we fix notations and recall several notions and facts. Let $M$ be an $n$-dimensional $(n \geq 2)$ smooth Riemannian manifold with a pole $p$ which is rotationally symmetric at $p$ (see [3]). Then the Riemannian metric in terms of geodesic polar coordinates at $p$ is given by

$$
d s^{2}=d r^{2}+f(r)^{2} d \Theta^{2},
$$

where $d \Theta^{2}$ is the standard metric of the unit sphere $S^{n-1}$ and $f$ is a nonnegative smooth function on $[0, \infty)$ such that $f>0$ in $(0, \infty), f(0)=0, f^{\prime}(0)=1$ and $f^{\prime \prime}(0)=0$. The Laplace-Beltrami operator $\Delta$ on $M$ is represented in the polar coordinates by

$$
\Delta=f^{1-n} \partial / \partial r\left(f^{n-1} \partial / \partial r\right)+f^{-2} \Lambda,
$$

where $\Lambda$ is the standard Laplace-Beltrami operator on $S^{n-1}$. The Riemannian measure $\nu$ of $M$ is given by $d \nu=f^{n-1}(r) d r d \sigma$, where $d \sigma$ is the standard Riemannian volume element on $S^{n-1}$. In what follows, we shall identify $M$ and the pole $p$ with $\mathbb{R}^{n}$ and the origin 0 of $\mathbb{R}^{n}$, respectively.

Let $G$ be the Green function of $-\Delta+1$ on $M$. In our setting, the notion of $[\mathrm{SSP}]$ is stated as follows.

[SSP] For any $\epsilon>0$ there exists a compact subset $K$ of $M$ such that

$$
\int_{M \backslash K} G(0, z) G(z, y) d \nu(z) \leq \epsilon G(0, y), \quad y \in M \backslash K .
$$

It is well-known (see, for example, [18]) that [SSP] implies the following condition $[\mathrm{NUP}]$ (non-uniqueness for the positive Cauchy problem).

[NUP] The Cauchy problem

$$
\partial_{t} u=\Delta u \text { in } M \times(0, T), \quad u(x, 0)=0 \text { on } M
$$

admits a non-zero nonnegative solution.

We say [UP] (uniqueness for the positive Cauchy problem) holds for (1.4) when any nonnegative solution of (1.4) is identically zero. Let us recall that when [UP] holds for (1.4), the structure of all nonnegative solutions to (1.1) is extremely simple. Namely, the following theorem holds (see [1]). 
Fact AT. Assume [UP]. Then, for any nonnegative solution $u$ of (1.1), there exists a unique Borel measure $\mu$ on $M$ such that

$$
u(x, t)=\int_{M} p(x, y, t) d \mu(y), \quad(x, t) \in M \times(0, T),
$$

where $p(x, y, t)$ is the minimal fundamental solution for (1.1). Conversely for any Borel measure $\mu$ on $M$ satisfying

$$
\int_{M} p(0, y, t) d \mu(y)<\infty, \quad 0<t<T
$$

the right hand side of (1.5) is a nonnegative solution of (1.1).

We denote by $S(r)$ and $B(r), r>0$, the geodesic sphere and ball with center 0 and radius $r$, respectively. The volume of $B(r)$ and the area of $S(r)$ are denoted by $|B(r)|$ and $|S(r)|$, respectively:

$$
|B(r)|=\sigma_{n} \int_{0}^{r} f(s)^{n-1} d s, \quad|S(r)|=\sigma_{n} f(r)^{n-1},
$$

where $\sigma_{n}$ is the area of the standard unit sphere $S^{n-1}$. The volume of the exterior of $B(r)$ is denoted by $|M \backslash B(r)|$.

Now, we are ready to state our main results. We first give a necessary and sufficient condition for [SSP].

Theorem 1.1 The condition [SSP] holds if and only if either the following condition (I) or (II) holds:

$$
\text { (I) } \int_{1}^{\infty} \frac{|M \backslash B(r)|}{|S(r)|} d r<\infty ; \quad \text { (II) } \int_{1}^{\infty} \frac{|B(r)|}{|S(r)|} d r<\infty .
$$

This theorem is proved in Section 2.

Note that (I) is equivalent to

$$
\int_{1}^{\infty} f(s)^{1-n}\left(\int_{s}^{\infty} f(r)^{n-1} d r\right) d s<\infty
$$

or

$$
\int_{1}^{\infty} f(r)^{n-1}\left(\int_{1}^{r} f(s)^{1-n} d s\right) d r<\infty .
$$

Similarly, (II) is equivalent to

$$
\int_{1}^{\infty} f(r)^{n-1}\left(\int_{r}^{\infty} f(s)^{1-n} d s\right) d r<\infty .
$$


It is well-known that $-\Delta$ on $M$ is subcritical (i.e., there exists a positive Green function of $-\Delta$ on $M$ ) if and only if

$$
\int_{1}^{\infty} f(s)^{1-n} d s<\infty
$$

(see Proposition 3.1 of [5], and also [13]). Thus, in the case (II), $-\Delta$ on $M$ is subcritical. On the other hand, in the case (I), $-\Delta$ on $M$ is critical (i.e., there exists a positive solution of $-\Delta u=0$ in $M$ but there exists no positive Green function of $-\Delta$ on $M$ ). Indeed (1.7) yields

$$
\int_{1}^{\infty} f(r)^{n-1} d r<\infty
$$

which together with the Schwarz inequality implies $\int_{1}^{\infty} f(r)^{1-n} d r=\infty$.

A curious feature of the characterization of [SSP] in Theorem 1.1 is that the family of manifolds satisfying [SSP] consists of two extremal cases (I) and (II). Roughly speaking, a manifold satisfying (I) or (II) shrinks or grows very rapidly at infinity, respectively, as illustrated by Example 1.6 below. The condition (II) is known to be a necessary and sufficient condition for the explosion of the Brownian motion on $M$ (see [5] and Subsection 4.1 below). The condition (I), however, is new. Since a manifold satisfying (I) is close to a compact manifold, it is quite natural that it satisfies the condition [SSP]. But, surprisingly, conditions similar to (I) seem not to have appeared in the literature.

We denote by $\partial_{M} M$ and $\partial_{m} M$ the Martin boundary and minimal Martin boundary of $M$ for $-\Delta+1$, respectively. We next determine $\partial_{M} M$ in the cases (I) and (II).

Theorem $1.2(i)$ Suppose that (I) holds. Then $\partial_{M} M=\partial_{m} M=\{\infty\}$, where $\infty$ is the point at infinity.

(ii) Suppose that (II) holds. Then $\partial_{M} M=\partial_{m} M=\infty S^{n-1}$, where $\infty S^{n-1}$ is the sphere at infinity.

This theorem is proved in Section 3.

In order to state an integral representation theorem for nonnegative solutions of (1.1) under the condition [SSP], we recall several facts. Let $Q$ be the quadratic form on $C_{0}^{\infty}(M)$ defined by $Q[u]=\int_{M}|\nabla u|^{2} d \nu$. Denote by $[-\Delta]_{M}$ the selfadjoint operator in $L^{2}(M ; d \nu)$ associated with the closure of $Q$. It is known ( $[19])$ that $[-\Delta]_{M}$ admits a complete orthonormal base of eigenfunctions $\left\{\phi_{j}\right\}_{j=0}^{\infty}$ with eigenvalues $\lambda_{0}<\lambda_{1} \leq \lambda_{2} \leq \cdots$ repeated according to multiplicity, where the smallest eigenvalue $\lambda_{0}$ is of multiplicity 1 and the eigenfunction $\phi_{0}$ may and will be assumed to be positive on $M$. 
We see that in the case (I),

$$
\lambda_{0}=0 \quad \text { and } \quad \phi_{0}=|M|^{-1 / 2},
$$

where $|M|=\sigma_{n} \int_{0}^{\infty} f(s)^{n-1} d s$ is the volume of $M$. In the case (II),

$$
\lambda_{0}>0 \text { and } \phi_{0} \text { is a radially symmetric function, }
$$

since $\lambda_{0}$ is of multiplicity one and $\Delta$ is invariant under rotation. Furthermore, since the Green function $g(0, x)$ (with pole 0 ) of $-\Delta$ on $M$ is given by

$$
g(0, x)=\int_{|x|}^{\infty} \frac{d r}{|S(r)|},
$$

and $\phi_{0}(x)$ is comparable with $g(0, x)$ near infinity (see Theorem 1.5 of [16]),

$$
\phi_{0}(x) \approx \int_{|x|}^{\infty} f(s)^{1-n} d s .
$$

Here and in what follows, for any positive functions $g(r)$ and $h(r), g \approx h$ means that there exists a constant $C>1$ such that

$$
C^{-1} h(r) \leq g(r) \leq C h(r)
$$

for $r$ sufficiently large.

Combining Theorem 1.2 of [11], Theorems 1.1 and 1.2 above, we get the following theorem except for the last assertion.

Theorem 1.3 Assume the condition (I) or (II). Then, for any $\xi \in \partial_{M} M$ there exists the limit

$$
\lim _{M \ni y \rightarrow \xi} \frac{p(x, y, t)}{\phi_{0}(y)} \equiv q(x, \xi, t), \quad x \in M, t \in \mathbb{R} .
$$

Here, as functions of $(x, t),\left\{p(x, y, t) / \phi_{0}(y)\right\}_{y}$ converges to $q(x, \xi, t)$ as $y \rightarrow \xi$ uniformly on any compact subset of $M \times \mathbb{R}$. Furthermore, $q(x, \xi, t)$ is a continuous function on $M \times \partial_{M} M \times \mathbb{R}$ such that

$$
\begin{aligned}
& q>0 \quad \text { on } M \times \partial_{M} M \times(0, \infty), \\
& q=0 \quad \text { on } M \times \partial_{M} M \times(-\infty, 0], \\
& \left(\partial_{t}-\Delta\right) q(\cdot, \xi, \cdot)=0 \quad \text { on } M \times \mathbb{R} .
\end{aligned}
$$

Moreover, in the case $(\mathrm{I}), q(x, \xi, t)$ is a radially symmetric function of $x$; while in the case (II)

$$
q(x, O \xi, t)=q\left(O^{-1} x, \xi, t\right), \quad x \in M, \xi \in \partial_{M} M, t \in \mathbb{R},
$$

for any rotation $O$, where $O \xi=(\infty, O \omega)$ with $\xi=(\infty, \omega), \omega \in S^{n-1}$. 
Proof. We have only to show the last assertion. For any rotation $O$, we have $p(O x, O y, t)=p(x, y, t)$. In the case $(\mathrm{I})$, with $\xi=\infty$ we have

$$
q(O x, \infty, t)=q(x, \infty, t) .
$$

Thus, as a function of $x, q(x, \infty, t)$ is radially symmetric. In the case (II), we have

$$
q(O x, O \xi, t)=q(x, \xi, t)
$$

This implies (1.18).

Theorems 1.2 and 1.3 say that in the case (I) the minimal fundamental solution $p(x, y, t)$ itself has a positive limit as $y \rightarrow \infty$ :

$$
\lim _{M \ni y \rightarrow \infty} p(x, y, t)=q(x, \infty, t)>0, \quad x \in M, t>0 .
$$

This is quite interesting. In the one dimensional case, it is known among specialists (private communication by Matsuyo Tomisaki) that this property is related to the classification of the boundary point $\infty$. In the higher dimensional case, however, no one seems to have observed phenomena like this.

Combining Theorem 1.3 of [11], Theorems 1.1, 1.2 and 1.3 above, we get the following theorem.

Theorem 1.4 Assume the condition (I) or (II). Then, for any nonnegative solution $u$ of (1.1) there exists a unique pair of Borel measures $\mu$ on $M$ and $\lambda$ on $\partial_{M} M \times[0, T)$ such that

$$
u(x, t)=\int_{M} p(x, y, t) d \mu(y)+\int_{\partial_{M} M \times[0, t)} q(x, \xi, t-s) d \lambda(\xi, s)
$$

for any $(x, t) \in M \times(0, T)$.

Conversely, for any Borel measures $\mu$ on $M$ and $\lambda$ on $\partial_{M} M \times[0, T)$ such that

$$
\begin{gathered}
\int_{M} p(0, y, t) d \mu(y)<\infty, \quad 0<t<T, \\
\int_{\partial_{M} M \times[0, t)} q(0, \xi, t-s) d \lambda(\xi, s)<\infty, \quad 0<t<T,
\end{gathered}
$$

the right hand side of (1.19) is a nonnegative solution of (1.1).

Obviously, it follows from Theorem 1.4 that the condition (I) or (II) implies [NUP]. A natural problem is whether [UP] holds if neither (I) nor (II) is satisfied. This is a difficult problem in full generality. But we have a simple and optimal partial answer to this problem. 
Theorem 1.5 Suppose that there exist positive constants $C, R$ and positive continuous increasing function $\rho$ on $[R, \infty)$ such that

$$
\begin{gathered}
C^{-1} \leq \frac{f(r+s)}{f(r)} \leq C, \quad r \geq R+\frac{1}{\rho(R)},|s| \leq \frac{1}{\rho(r)}, \\
\int_{R}^{\infty} \frac{d r}{\rho(r)}=\infty .
\end{gathered}
$$

Then [UP] holds for (1.4).

This theorem is proved in Section 4.

As for uniqueness of bounded solutions for the Cauchy problem (1.4), see Fact NUB to be stated in Subsection 4.1.

Here let us examine the conditions in Theorems 1.1 and 1.5 by a simple example.

Example 1.6 For $\alpha>0$, put $\Psi_{ \pm}(r)=\exp \left( \pm r^{\alpha}\right)$. Let $f(r)=\Psi_{+}(r)$ or $\Psi_{-}(r)$ for $r>1$. Since $f^{\prime}(r) / f(r)= \pm \alpha r^{\alpha-1}$, the condition (1.22) is satisfied with $\rho(r)=\max \left(r^{\alpha-1}, 1\right)$ for $r>1$. Thus (1.23) is satisfied if $\alpha \leq 2$. On the other hand, for $\alpha>2, f=\Psi_{+}$or $\Psi_{-}$satisfies the condition (II) or (I), respectively. Summing up,

in the case (I), the manifold $M$ shrinks very rapidly at infinity;

in the case (II), $M$ grows very rapidly at infinity;

in the case $[\mathrm{UP}], M$ changes mildly near infinity.

The sectional curvature at a point $x=(r, \omega)$ of a plane in $T_{x} M$ containing $\partial / \partial r$ depends only on $r$, and is called a radial curvature. Denote it by $k(r)$. It is given by

$$
k(r)=-f^{\prime \prime}(r) / f(r), \quad r>0
$$

(see [3] and references therein).

Finally, under some conditions on $k(r)$ near infinity, we give a necessary and sufficient condition for [SSP] or [UP].

Theorem 1.7 Suppose that $-k(r)$ is positive and increasing on $[R, \infty)$ for some $R>0$. Assume

$$
\int_{R}^{\infty} \frac{\left|k^{\prime}(r)\right|}{|k(r)|}\left(\sup _{s \geq r} \frac{\left|k^{\prime}(s)\right|}{|k(s)|^{3 / 2}}\right) d r<\infty .
$$

Then $[\mathrm{SSP}]$ holds if and only if

$$
\int_{R}^{\infty}[-k(r)]^{-1 / 2} d r<\infty
$$


while $[\mathrm{UP}]$ holds if and only if

$$
\int_{R}^{\infty}[-k(r)]^{-1 / 2} d r=\infty
$$

This theorem is a direct consequence of Theorems 5.5 and 5.6 to be stated and proved in Section 5. As for results via curvature comparison techniques, see Theorems 5.1 and 5.4 to be given in Section 5 .

The remainder of this paper is organized as follows. Theorems 1.1 and 1.2 are proved in Sections 2 and 3, respectively. In Section 4 we study relations between [SSP] and uniqueness of bounded solutions to (1.4), and prove Theorem 1.5. In Section 5 we give sufficient conditions for [SSP] and [UP] in terms of the radial curvature.

\section{Semismall perturbations}

In this section we prove Theorem 1.1. Its proof is decomposed into three lemmas. Let $G$ be the Green function of $-\Delta+1$ on $M$. Put

$$
L_{1}=-f^{1-n} \frac{d}{d r}\left(f^{n-1} \frac{d}{d r}\right) .
$$

Let $H$ be the Green function of $L_{1}+1$ on $I=(0, \infty)$. We see that there exists a unique positive solution of the equation

$$
L_{1} h+h=0 \quad \text { in } I, \quad h(r)=1+o(1) \quad \text { as } r \rightarrow 0 .
$$

By Theorem 6.1 of [17] we have

$$
H(r, s)=\gamma h(r) g(s), \quad 0<r<s<\infty
$$

where $\gamma>0$ is a constant and $g(s)$ is another positive solution of the equation $L_{1} g+g=0$ in $I$, which is linearly independent of $h$ and behaves like $s^{2-n}$ or $\log 1 / s$ as $s \rightarrow 0$ for $n \geq 3$ or $n=2$. Thus there exists a positive limit $H(0, s)=\lim _{r \rightarrow 0} H(r, s)$. The following lemma is a key to the proof of Theorem 1.1.

Lemma 2.1 [SSP] holds if and only if 1 is a semismall perturbation of $L_{1}+1$ on $I=(0, \infty)$, i.e., for any $\epsilon>0$ there exists a compact subset $J$ of $(0, \infty)$ such that

$$
\int_{I \backslash J} H(1, r) H(r, s) f^{n-1}(r) d r \leq \epsilon H(1, s), \quad s \in I \backslash J .
$$


Proof. Note that the smallest eigenvalue of $-\Lambda$ on $S^{n-1}$ is 0 and $\psi_{0}=\sigma_{n}^{-1 / 2}$ is the corresponding normalized eigenfunction. By Lemma 8.1 of [17], which is shown by Fubini's theorem, we have

$$
\int_{S^{n-1}} G(r \omega, y) d \sigma(\omega)=H(r, s), \quad r>0, s=|y|>0,
$$

where $d \sigma$ is the area element of $S^{n-1}$. From this we have

$$
G(0, s \omega)=\sigma_{n}^{-1} H(0, s), \quad s>0, \omega \in S^{n-1} .
$$

Since $d \nu(z)=f^{n-1}(r) d r d \sigma(\omega)$ with $z=r \omega$, we get

$$
\int_{|z|>R} G(0, z) G(z, y) d \nu(z)=\int_{R}^{\infty} \sigma_{n}^{-1} H(0, r) H(r, s) f^{n-1}(r) d r
$$

for any $s=|y|>0$ and $R>0$. Since $H(1, r)$ and $H(0, r)$ are comparable on $(2, \infty)$, this together with $(2.3)$ shows that [SSP] holds if and only if for any $\epsilon>0$ there exists $R>2$ such that

$$
\int_{R}^{\infty} H(1, r) H(r, s) f^{n-1}(r) d r \leq \epsilon H(1, s)
$$

for any $s>R$. Note that if (2.5) holds for $s>R$, then (2.5) holds for any $s>0$. On the other hand, from (2.1) we obtain that for any $\epsilon>0$ there exists $\delta>0$

$$
\int_{0}^{\delta} H(1, r) H(r, s) f^{n-1}(r) d r \leq \epsilon H(1, s), s>0
$$

(see also Theorem 6.3 of [17] and Theorems 2.5 and 2.6 of [22]). This completes the proof of the lemma.

Lemma 2.2 Suppose that $L_{1}$ on $(0, \infty)$ is subcritical. Then 1 is a semismall perturbation of $L_{1}+1$ on $(0, \infty)$ if and only if (II) holds.

Proof. By Theorem 2.5 of [22], 1 is a semismall perturbation of $L_{1}+1$ on $(0, \infty)$ if and only if 1 is a semismall perturbation of $L_{1}$ on $(0, \infty)$, which is equivalent to the condition (II) (see also Theorem 6.3 of [17]).

Lemma 2.3 Suppose that $L_{1}$ on $(0, \infty)$ is critical. Then 1 is a semismall perturbation of $L_{1}+1$ on $(0, \infty)$ if and only if $(\mathrm{I})$ holds.

Proof. Choose a nonnegative function $\Psi \in C_{0}^{\infty}((0,1 / 2))$ which is not identically zero. Then, by Theorem 1.6 of [16], the Green functions of $L_{1}+1$ and $L_{1}+\Psi+1$ on $(0, \infty)$ are comparable. Thus, by Theorem 2.5 of [22], 1 is 
a semismall perturbation of $L_{1}+1$ on $(0, \infty)$ if and only if 1 is a semismall perturbation of $L_{1}+\Psi$ on $(0, \infty)$. By Theorem 1.1 of [13], $L_{1}$ on $(0, \infty)$ is critical if and only if

$$
\int_{1 / 2}^{\infty} f(s)^{1-n} d s=\infty .
$$

Let $K^{0}$ and $K^{\infty}$ be the Martin kernels for $L_{1}+\Psi$ at 0 and $\infty$, respectively. By Theorem 1.5 of [16] and Theorem 6.1 of [17], $K^{0}(r)$ is comparable to the constant function 1 on $(1, \infty)$. Furthermore, $K^{\infty}(r)$ is comparable to the function

$$
\int_{1 / 2}^{r} f(s)^{1-n} d s \quad \text { on }(1, \infty)
$$

Thus 1 is a semismall perturbation of $L_{1}+\Psi$ on $(0, \infty)$ if and only if

$$
\int_{1}^{\infty} 1 \cdot\left(\int_{1 / 2}^{r} f(s)^{1-n} d s\right) f(r)^{n-1} d r<\infty .
$$

This is equivalent to (I).

Remark 2.4 It is an open problem whether [SSP] for $-\Delta+1$ on $M$ implies [SP] ( 1 is a small perturbation of $-\Delta+1$ on $M)$, i.e., for any $\epsilon>0$ there exists a compact subset $K$ of $M$ such that

$$
\int_{M \backslash K} G(x, z) G(z, y) d \nu(z) \leq \epsilon G(x, y), \quad x, y \in M \backslash K .
$$

\section{Elliptic Martin boundaries}

In this section we prove Theorem 1.2. The proof is based upon the following proposition which can be shown in the same way as in the proof of Theorems 3.2 and 3.3 of [12] (see also Theorem 1.3 of [13], Theorem 6.3 and Example 10.1 of [17]).

Proposition 3.1 (i) Suppose that (I) holds. Then $\partial_{M} M=\partial_{m} M=\{\infty\}$ if and only if

$$
\int_{1}^{\infty} f(r)^{n-3}\left(\int_{1 / 2}^{r} f(s)^{1-n} d s\right) d r=\infty .
$$

(ii) Suppose that (II) holds. Then $\partial_{M} M=\partial_{m} M=\infty S^{n-1}$ if and only if

$$
\int_{1}^{\infty} f(r)^{n-3}\left(\int_{r}^{\infty} f(s)^{1-n} d s\right) d r<\infty .
$$


We first treat the case (I).

Lemma 3.2 If (I) holds, then (3.1) holds true.

Proof. Put

$$
g(r)=\int_{1 / 2}^{r} f(s)^{1-n} d s, \quad J=\int_{1}^{\infty} f(r)^{n-3} g(r) d r .
$$

We see that (I) implies $\lim _{r \rightarrow \infty} g(r)=\infty$. When $n=2$, we have

$$
J=\int_{1}^{\infty} f(r)^{-1} g(r) d r \geq g(1) \int_{1}^{\infty} f(r)^{1-n} d r=\infty .
$$

When $n=3$, we have

$$
J=\int_{1}^{\infty} g(r) d r \geq g(1) \int_{1}^{\infty} d r=\infty .
$$

Let $n \geq 4$. Since $g^{\prime}(r)=f(r)^{1-n}$, we have

$$
J=\int_{1}^{\infty} g(r)\left[g^{\prime}(r)\right]^{-(n-3) /(n-1)} d r .
$$

Put $\Omega=\left\{r>1: g(r)<g^{\prime}(r)\right\}$. We claim that the Lebesgue measure $|\Omega|$ of $\Omega$ is infinite. Suppose that $|\Omega|<\infty$. Since (I) is equivalent to (2.7), we have

$$
\infty>\int_{1}^{\infty} g(r) / g^{\prime}(r) d r \geq \int_{(1, \infty) \backslash \Omega} d r=\infty
$$

This is a contradiction. We have

$$
J \geq \int_{\Omega}\left[\frac{g(r)}{g^{\prime}(r)}\right]^{(n-3) /(n-1)} g(r)^{2 /(n-1)} d r \geq \int_{\Omega} \frac{g(r)}{g^{\prime}(r)} \cdot g(r)^{2 /(n-1)} d r .
$$

On the other hand,

$$
\begin{aligned}
\infty & =|\Omega| \\
& =\int_{\Omega}\left[g(r)^{1+2 /(n-1)} / g^{\prime}(r)\right]^{1 / 2}\left[g^{\prime}(r) / g(r)^{1+2 /(n-1)}\right]^{1 / 2} d r \\
& \leq\left\{\int_{\Omega}\left[g(r)^{1+2 /(n-1)} / g^{\prime}(r)\right] d r\right\}^{1 / 2}\left\{\int_{\Omega}\left[g^{\prime}(r) / g(r)^{1+2 /(n-1)}\right] d r\right\}^{1 / 2} .
\end{aligned}
$$

But

$$
\begin{aligned}
\int_{\Omega} g^{\prime}(r) g(r)^{-1-2 /(n-1)} d r & \leq \int_{1}^{\infty} g^{\prime}(r) g(r)^{-1-2 /(n-1)} d r \\
& =\left[-\frac{n-1}{2} g(r)^{-2 /(n-1)}\right]_{1}^{\infty}=\frac{n-1}{2} g(1)^{-2 /(n-1)}<\infty .
\end{aligned}
$$


Thus

$$
\int_{\Omega}\left[g(r)^{1+2 /(n-1)} / g^{\prime}(r)\right] d r=\infty .
$$

This together with (3.4) implies (3.1). This completes the proof of the lemma.

Next we treat the case (II).

Lemma 3.3 If (II) holds, then (3.2) holds.

Proof. Put

$$
F(r)=\int_{r}^{\infty} f(s)^{1-n} d s, \quad I=\int_{1}^{\infty} f(r)^{n-3} F(r) d r .
$$

When $n=2$, we have

$$
I=\int_{1}^{\infty} f(r)^{1-n} F(r) d r \leq F(1) \int_{1}^{\infty} f(r)^{1-n} d r<\infty .
$$

Let $n \geq 3$. With $p=n-1$ and $q=(n-1) /(n-2)$, we have

$$
\begin{aligned}
I & =\int_{1}^{\infty} f(r)^{(1-n) / p+(n-1) / q} F(r) d r \\
& \leq\left(\int_{1}^{\infty} f(r)^{1-n} F(r) d r\right)^{1 / p}\left(\int_{1}^{\infty} f(r)^{n-1} F(r) d r\right)^{1 / q}<\infty .
\end{aligned}
$$

This completes the proof of the lemma.

Theorem 1.2 follows from Proposition 3.1, Lemmas 3.2 and 3.3.

\section{Uniqueness theorems}

\subsection{Uniqueness of bounded solutions}

In this subsection we describe relations between [SSP] and uniqueness of bounded solutions to the heat equation. We consider the following condition [NUB] (non-uniqueness of bounded solutions).

[NUB] The Cauchy problem

$$
\left(\partial_{t}-\Delta\right) u=0 \text { in } M \times(0, T), \quad u(x, 0)=0 \text { on } M
$$

admits a non-zero bounded solution. 
We say that [UB] (uniqueness of bounded solutions) holds when any bounded solution of (4.1) is identically zero. For [NUB], the following fact is wellknown (see Proposition 3.2 and Theorem 6.2 of [5], and also Example 3.15 of $[6])$.

Fact NUB. The condition [NUB] holds if and only if the condition (II) holds. In another word, [UB] holds if and only if (II) does not hold, i.e.,

$$
\int_{1}^{\infty} \frac{|B(r)|}{|S(r)|} d r=\infty
$$

Now, let us consider the case (I). In this case, by Theorem 1.2(i), there exists a positive solution $h$ of $(-\Delta+1) h=0$ in $M$, which is radial and unique up to a constant multiple. Furthermore, we see from the proof of Lemma 2.3 that $h(x)$ is comparable with

$$
g(|x|)=\int_{1 / 2}^{|x|} f(s)^{1-n} d s \quad \text { on }\{|x|>1\} .
$$

By Theorem 1.1 and Proposition 3.3 of [16], we have

$$
\int_{M} G(0, y) h(y) d \nu(y)<\infty .
$$

(This can be shown directly from (2.7).) It is well known that (4.3) yields the existence of a positive solution $u$ of the Cauchy problem

$$
\partial_{t} u=(\Delta-1) u \quad \text { in } M \times(0, \infty), \quad u(x, 0)=0 \quad \text { on } M
$$

such that $0<u(x, t)<h(x)$ in $M \times(0, \infty)$ (for more results, see Theorem 2.1 of [18] and references therein). Hence (I) implies the existence of a positive solution $v$ of the Cauchy problem (4.1) such that $0<v(x, t)<e^{t} h(x)$ in $M \times(0, \infty)$. Summing up, we have shown the following proposition.

Proposition 4.1 Suppose that (I) holds. Then [UB] holds true, but there exists a solution $v$ of (4.1) such that $0<v(x, t)<e^{t} h(x)$ in $M \times(0, \infty)$, where $h$ is a positive radial solution of $(-\Delta+1) h=0$ in $M$.

Here let us show that $h(x)$ grows as $|x| \rightarrow \infty$, at least, as fast as $\exp \left(C|x|^{2}\right)$ for some constant $C>0$. Put

$$
g(r)=\int_{1 / 2}^{r} f(s)^{1-n} d s
$$


For $r>2$, we have

$$
\begin{aligned}
\frac{r}{2} \leq r-1 & =\int_{1}^{r}\left[\frac{g(s)}{g^{\prime}(s)}\right]^{1 / 2}\left[\frac{g^{\prime}(s)}{g(s)}\right]^{1 / 2} d s \\
& \leq\left\{\int_{1}^{\infty} \frac{g(s)}{g^{\prime}(s)} d s\right\}^{1 / 2}\left\{\int_{1}^{r} \frac{g^{\prime}(s)}{g(s)} d s\right\}^{1 / 2}
\end{aligned}
$$

Thus $g(r) \geq g(1) \exp \left(B r^{2}\right)$ for some constant $B>0$, which implies

$$
h(x) \geq A \exp \left(B|x|^{2}\right), \quad x \in M,
$$

for some constant $A>0$.

\subsection{Uniqueness of nonnegative solutions}

In this subsection we prove Theorem 1.5.

Proof of Theorem 1.5. In [9] we introduced the notion of the parabolic Harnack principle with scale function $\rho([\mathrm{PHP}-\rho])$. In the setting of this paper, $[\mathrm{PHP}-\rho]$ reads as follows: there exists a positive constant $C_{p}$ such that for any $t \in[0, T), x \in B(0, S)=B(S), S>R+1 / \rho(R), 0<s \leq 1 / \rho(S)$, any nonnegative solution $u$ of the heat equation

$$
\partial_{t} u=\Delta u \quad \text { in } Q=B(x, s) \times\left(t-s^{2}, t+s^{2}\right)
$$

satisfies

$$
\sup _{Q_{-}} u \leq C_{p} \inf _{Q_{+}} u
$$

where

$Q_{-}=B\left(x, \frac{s}{2}\right) \times\left(t-\frac{3}{4} s^{2}, t-\frac{1}{4} s^{2}\right), \quad Q_{+}=B\left(x, \frac{s}{2}\right) \times\left(t+\frac{1}{4} s^{2}, t+\frac{3}{4} s^{2}\right)$.

Theorem 2.2 of [9] in our case says that if [PHP- $\rho$ ] holds with $\rho$ satisfying (1.23), then [UP] holds. Thus we have only to show [PHP-C $\rho$ ] for some large positive constant $C$. We see by (1.22) that uniformly in $x \in$ $B(0, S) \backslash B(0, R+1 / \rho(R))$ and $0<s \leq 1 / \rho(S)$, the subset $\{y \in M$ : $|x|-s<|y|<|x|+s\}$ is quasi-isometric to a piece of flat cylinder (see descriptions before Proposition 4.10 of [7]). Hence [PHP-C $\rho$ ] holds for some $C>0$, since the parabolic Harnack inequality is stable under quasiisometry (see [4], [8], [20], [21]). 


\section{Curvature conditions}

In this section we give sufficient conditions for [SSP] and [UP] in terms of the radial curvature $k(r), r>0$, which is the sectional curvature at a point $x=(r, \omega)$ of any plane in $T_{x} M$ containing $\partial / \partial r$. It is well-known that $k(r)$ satisfies the equation $f^{\prime \prime}+k f=0$ in $(0, \infty)$ (see [3] and references therein).

\subsection{Comparison of curvatures}

In this subsection we give sufficient conditions for [UP] and [SSP] via curvature comparison techniques. First we give a sufficient condition for [UP].

Theorem 5.1 Suppose that

$$
-K(r) \leq k(r) \leq 0, \quad r>0,
$$

for some positive continuous increasing function $K(r)$ on $[0, \infty)$. If

$$
\int_{1}^{\infty} \frac{d r}{\sqrt{K(r)}}=\infty
$$

then [UP] holds. Thus Fact UP holds true.

For proving this theorem, we need elementary lemmas.

Lemma 5.2 Suppose that $g, h \in C^{2}([0, \infty)), g(0)=h(0)=0, g^{\prime}(0)=$ $h^{\prime}(0)=1, g>0$ and $h>0$ in $(0, \infty)$. If $g^{\prime \prime} / g \leq h^{\prime \prime} / h$ in $(0, \infty)$, then $g \leq h$ and $g^{\prime} / g \leq h^{\prime} / h$ in $(0, \infty)$.

This is Lemma 3 of [10], and can be shown by calculating $\left(h^{2}(g / h)^{\prime}\right)^{\prime}$.

Let $h$ be a solution of the initial value problem

$$
h^{\prime \prime}(r)=K(r) h(r) \text { in }(0, \infty), \quad h(0)=0, \quad h^{\prime}(0)=1 .
$$

Then we see that $h>0$ and $h^{\prime}>0$ in $(0, \infty)$. Furthermore, we have the following lemma.

Lemma 5.3 There exists a positive constant $C$ such that

$$
\frac{h^{\prime}(r)}{h(r)} \leq C \sqrt{K(r)}, \quad r \geq 1 .
$$

Proof. This is nothing but the assertion (3.3) of Lemma 3.1 of [14]. But we give a proof, since the proof is simple and Lemma 3.1 is stated under a condition different from ours. 
Put $H=h^{\prime} / h$ and $G=H / \sqrt{K}$. Let $r>s \geq 1$. Since $H^{\prime}+H^{2}=K$,

$$
H(r)-H(s)=\int_{s}^{r} K(t)\left[1-G(t)^{2}\right] d t
$$

Since $K$ is increasing, we have

$$
G(r)-G(s) \leq \int_{s}^{r} \sqrt{K(t)}\left[1-G(t)^{2}\right] d t
$$

We claim that

$$
G(r) \leq M \equiv \max (1, G(1)) \text { for } r \geq 1
$$

On the contrary, suppose that there exists $r>1$ such that $G(r)>M$. Put

$$
S=\inf \{s \in[1, r] ; G(t) \geq G(r) \text { for any } t \text { in }[s, r]\} .
$$

Then, by (5.5), $G(S)>G(r)$. Thus $S=1$. This yields $G(1)>G(r)>G(1)$, which is a contradiction.

Proof of Theorem 5.1. We have

$$
f^{\prime \prime}(r)=-k(r) f(r) \text { in }(0, \infty), \quad f(0)=0, \quad f^{\prime}(0)=1,
$$

$f>0$ and $f^{\prime}>0$ in $(0, \infty)$. By Lemma 5.2, $f \leq h$ and $0<f^{\prime} / f \leq h^{\prime} / h$ in $(0, \infty)$. By Lemma 5.3,

$$
\left(f^{\prime}(r) / f(r)\right)^{2} \leq C^{2} K(r), \quad r \geq 1 .
$$

For a point $x=(r, \omega) \in M, r>1$, and a plane $\pi$ in $T_{x} M$, denote by $\sec (\pi)$ the sectional curvature of $\pi$. By the formula on p.27 of [2] (or see (4.3) of [14]),

$$
\sec (\pi) \geq-\left(\frac{f^{\prime}(r)}{f(r)}\right)^{2}-\frac{f^{\prime \prime}(r)}{f(r)}
$$

Thus, for any plane $\pi$ in $T_{(r, \omega)} M$,

$$
\sec (\pi) \geq-C K(r), \quad r>1,
$$

for some positive constant $C$ independent of $r$.

By virtue of Example 1.5 of [9] (which is an improved version of Theorem A of [14]), the lower bound (5.9) together with the assumption (5.2) shows the theorem. 
We can also show Theorem 5.1 via Theorem 1.5.

The second proof of Theorem 5.1. Choose a positive constant $C$ such that $C \sqrt{K(1)} \geq 1$ and $f^{\prime}(r) / f(r) \leq C \sqrt{K(r)}$ for $r \geq 1$ (see (5.7)). Put $\rho(r)=C K(r+1)^{1 / 2}$. Then we claim that $(1.22)$ is satisfied with $R=1$. Let $r \geq 1+1 / \rho(1)$. For $0 \leq s \leq 1 / \rho(r)$, we have

$$
0 \leq \log \frac{f(r+s)}{f(r)}=\int_{r}^{r+s} \frac{f^{\prime}(t)}{f(t)} d t \leq \rho(r) s \leq 1
$$

Thus $1 \leq f(r+s) / f(r) \leq e$. Similarly,

$$
1 \leq f(r) / f(r+s) \leq e, \quad-1 / \rho(r) \leq s \leq 0
$$

Thus (1.22) is satisfied with $R=1$. Hence, by Theorem 1.5, [UP] holds.

Next, we give a sufficient condition for (II), the second case of [SSP]. Let $\kappa(r)$ be a continuous increasing function on $[0, \infty)$ such that $\kappa(R)>0$ for some $R>0$. Let $g$ be a solution of the initial value problem

$$
g^{\prime \prime}(r)=\kappa(r) g(r) \text { in }(0, \infty), \quad g(0)=0, \quad g^{\prime}(0)=1 .
$$

Assume that $g$ satisfies

$$
g>0 \text { in }(0, \infty), \quad \lim _{r \rightarrow \infty} g(r)=\infty
$$

Obviously, (5.11) is satisfied if $\kappa \geq 0$ on $[0, \infty)$.

Theorem 5.4 Let $\kappa(r)$ be a function as above for which the solution $g$ of (5.10) satisfies (5.11). Suppose that

$$
k(r) \leq-\kappa(r), \quad r>0 .
$$

If

$$
\int_{R}^{\infty} \frac{d r}{\sqrt{\kappa(r)}}<\infty
$$

then the condition (II) holds true. Thus Theorems 1.3 and 1.4 hold.

Proof. It follows from the proof of Theorem B of [14] that the condition [NUB] (see Section 4) holds. Thus, by Fact NUB, the condition (II) holds. 


\subsection{Radial curvature conditions near infinity}

In this subsection we treat a class of rotationally symmetric Riemannian manifolds whose radial curvatures satisfy only conditions near infinity, and give sufficient conditions for [SSP] and [UP].

Let $k(r)$ be the radial curvature, and put $q(r)=-k(r)$. Assume that $k(r)$ satisfies the following conditions:

(k-1) There exists $R>0$ such that $k<0$ on $[R, \infty)$.

(k-2) $\quad \int_{R}^{\infty} q^{1 / 2}(r) d r=\infty$.

(k-3) $\quad \int_{R}^{\infty} \frac{\left|q^{\prime}(s)\right|}{q(s)}\left(\sup _{r \geq s} \frac{\left|q^{\prime}(r)\right|}{q^{3 / 2}(r)}\right) d s<\infty$.

Under these conditions we have the following theorems.

Theorem 5.5 [SSP] holds if

$$
\int_{R}^{\infty} q^{-1 / 2}(r) d r<\infty
$$

Theorem 5.6 [UP] holds if

$$
\int_{R}^{\infty}\left(\sup _{R \leq s \leq r} q(s)\right)^{-1 / 2} d r=\infty .
$$

Before we give proofs of Theorems 5.5 and 5.6, we see a simple example.

Example 5.7 Suppose that $k(r)=-r^{\alpha}$ on $[1, \infty)$, where $\alpha>-2$. Then the conditions (k-1), (k-2) and (k-3) are satisfied. Furthermore, (5.14) is satisfied if and only if $\alpha>2$.

Now, consider the equation

$$
y^{\prime \prime}=q(r) y \quad \text { in }(R, \infty) .
$$

A special case of Lemma 4.3 of [15] yields the following lemma.

Lemma 5.8 The equation (5.16) has solutions $y_{+}$and $y_{-}$such that

$$
\begin{aligned}
& y_{ \pm}=q^{-1 / 4} e^{ \pm \theta_{0}}\left(1+h_{ \pm}\right), \\
& y_{ \pm}^{\prime}= \pm q^{1 / 4} e^{ \pm \theta_{0}}\left(1+\tilde{h}_{ \pm}\right),
\end{aligned}
$$

where $\lim _{r \rightarrow \infty} h_{ \pm}(r)=0=\lim _{r \rightarrow \infty} \tilde{h}_{ \pm}(r)$ and

$$
\theta_{0}(r)=\int_{R}^{r} q^{1 / 2}(s) d s .
$$


Here we note that for any $0<c<1$

$$
\begin{gathered}
y_{+}(r) \geq c \exp \left[c \int_{R}^{r} q^{1 / 2}(s) d s\right], \\
y_{-}(r) \leq(1+c) \exp \left[-c \int_{R}^{r} q^{1 / 2}(s) d s\right]
\end{gathered}
$$

if $r$ is sufficiently large. Let us show only (5.20). Put

$$
Q(r)=\int_{R}^{r} q^{1 / 2}(s) d s-\frac{1}{4} \log q(r) .
$$

Then

$$
Q^{\prime}(r)=q^{1 / 2}(r)-\frac{1}{4} \frac{q^{\prime}(r)}{q(r)}=q^{1 / 2}(r)\left\{1-\frac{1}{4} \frac{q^{\prime}(r)}{q^{3 / 2}(r)}\right\} .
$$

Put $R(s)=\sup _{r \geq s}\left|q^{\prime}(r)\right| / q^{3 / 2}(r)$. In the case $\lim _{s \rightarrow \infty} R(s)=0$,

$$
Q^{\prime}(r)=q^{1 / 2}(r)(1+o(1)) \quad \text { as } r \rightarrow \infty ;
$$

while in the case $\lim _{s \rightarrow \infty} R(s)>0$, (k-3) yields

$$
\int_{R}^{\infty} \frac{\left|q^{\prime}(s)\right|}{q(s)} d s<\infty .
$$

Thus, in both cases, we get (5.20) by $(\mathrm{k}-2)$ and $(\mathrm{k}-3)$.

By Lemma 5.8, there exist constants $C_{1}$ and $C_{2}$ such that

$$
f(r)=C_{1} y_{+}(r)+C_{2} y_{-}(r) \quad \text { in }(R, \infty) .
$$

Since $f>0$ in $(0, \infty)$, there are only two possibilities: the growth case $[G]$ where $C_{1}>0$, and the decay case [D] where $C_{1}=0$ and $C_{2}>0$.

In case $[\mathrm{G}]$, as $r \rightarrow \infty$

$$
\begin{aligned}
f(r) & =C_{1} q^{-1 / 4}(r) e^{\theta_{0}(r)}(1+o(1)), \\
f^{\prime}(r) & =C_{1} q^{1 / 4}(r) e^{\theta_{0}(r)}(1+o(1)) .
\end{aligned}
$$

In case $[\mathrm{D}]$, as $r \rightarrow \infty$

$$
\begin{aligned}
f(r) & =C_{2} q^{-1 / 4}(r) e^{-\theta_{0}(r)}(1+o(1)), \\
f^{\prime}(r) & =-C_{2} q^{1 / 4}(r) e^{-\theta_{0}(r)}(1+o(1)) .
\end{aligned}
$$

We first show Theorem 5.5. The following lemma completes the proof of Theorem 5.5.

Lemma 5.9 Assume (5.14). (i) In case [G], condition (II) is satisfied. (ii) In case $[\mathrm{D}]$, condition $(\mathrm{I})$ is satisfied. 
Recall that for any positive functions $g(r)$ and $h(r)$ defined for $r$ sufficiently large, $g \approx h$ means that there exists a constant $C>1$ such that

$$
C^{-1} h(r) \leq g(r) \leq C h(r)
$$

for $r$ sufficiently large.

Proof of Lemma 5.9. The case [G]. First let $n=2$. By (5.23) and (5.18),

$$
\int_{r}^{\infty} f(s)^{1-n} d s \approx \int_{r}^{\infty}\left[-y_{-}^{\prime}(s)\right] d s=y_{-}(r) .
$$

Thus

$$
f(r)^{n-1} \int_{r}^{\infty} f(s)^{1-n} d s \approx q^{-1 / 2}(r) .
$$

Hence (II) holds true. Next, let $n \geq 3$.

$$
\begin{aligned}
\int_{r}^{\infty} f(s)^{1-n} d s & \approx \int_{r}^{\infty}\left(-y_{-}^{\prime}(s)\right) y_{+}(s)^{2-n} d s \\
& =y_{-}(r) y_{+}(r)^{2-n}+\int_{r}^{\infty} y_{-}(s)(2-n) y_{+}(s)^{1-n} y_{+}^{\prime}(s) d s .
\end{aligned}
$$

Since $y_{-}(s) y_{+}^{\prime}(s) \approx 1$, we have

$$
\int_{r}^{\infty} f(s)^{1-n} d s \approx y_{-}(r) y_{+}(r)^{2-n} .
$$

Thus

$$
f(r)^{n-1} \int_{r}^{\infty} f(s)^{1-n} d s \approx y_{+}(r)^{n-1} y_{-}(r) y_{+}(r)^{2-n} \approx q^{-1 / 2}(r) .
$$

Hence (II) holds true.

The case [D]. First let $n=2$. By (5.25) and (5.18),

$$
\int_{1 / 2}^{r} f(s)^{1-n} d s \approx \int_{1}^{r} y_{+}^{\prime}(s) d s \approx y_{+}(r) .
$$

Thus

$$
f(r)^{n-1} \int_{1 / 2}^{r} f(s)^{1-n} d s \approx q^{-1 / 2}(r) .
$$

Hence (I) holds true. Next, let $n \geq 3$.

$$
\begin{aligned}
\int_{1 / 2}^{r} f(s)^{1-n} d s & \approx \int_{1 / 2}^{r} y_{+}^{\prime}(s) y_{-}(s)^{2-n} d s \\
& =y_{+}(r) y_{-}(r)^{2-n}-\int_{1 / 2}^{r} y_{+}(s)(2-n) y_{-}(s)^{1-n} y_{-}^{\prime}(s) d s .
\end{aligned}
$$


Since $-y_{+}(s) y_{-}^{\prime}(s) \approx 1$, we have

$$
\int_{1 / 2}^{r} f(s)^{1-n} d s \approx y_{+}(r) y_{-}(r)^{2-n}
$$

Thus

$$
f(r)^{n-1} \int_{1 / 2}^{r} f(s)^{1-n} d s \approx y_{-}(r)^{n-1} y_{+}(r) y_{-}(r)^{2-n} \approx q^{-1 / 2}(r) .
$$

Hence (I) holds true.

We next show Theorem 5.6. Put $\tau=\sup _{r \geq R}\left|q^{\prime}(r)\right| / q^{3 / 2}(r)$, which is finite by $(\mathrm{k}-3)$, and define $\rho$ by

$$
\rho(r)=\tau\left(\sup _{R \leq s \leq r} q(s)\right)^{1 / 2}, \quad r \geq R
$$

and $\rho(r)=\tau q^{1 / 2}(R)$ for $0 \leq r \leq R$. Then we have

Lemma 5.10 For $r \geq R+1 / \rho(R)$ and $s$ with $|s| \leq 1 / \rho(r)$,

$$
\frac{2}{3} \leq \frac{q^{1 / 2}(r+s)}{q^{1 / 2}(r)} \leq 2
$$

Proof. We have

$$
\left|\left(\frac{1}{q^{1 / 2}(t)}\right)^{\prime}\right|=\frac{1}{2} \cdot \frac{\left|q^{\prime}(t)\right|}{q^{3 / 2}(t)} \leq \frac{\tau}{2}
$$

Thus

$$
\left|\frac{q^{1 / 2}(r)}{q^{1 / 2}(r+s)}-1\right| \leq \frac{\tau}{2}|s| q^{1 / 2}(r) \leq \frac{1}{2} .
$$

This implies (5.27).

Lemma 5.11 Assume (5.15). Then [UP] holds true.

Proof. By (5.17), (5.18) and (5.23)-(5.26),

$$
\frac{f^{\prime}(r)}{f(r)} \approx q^{1 / 2}(r) .
$$

By Lemma 5.10, the condition (1.22) is satisfied. Hence Theorem 1.5 together with the assumption (5.15) shows the lemma.

This lemma completes the proof of Theorem 5.6. 


\section{References}

[1] Ancona, A. And TAYlor, J. C.: Some remarks on Widder's theorem and uniqueness of isolated singularities for parabolic equations. In Partial differential equations with minimal smoothness and applications, 15-23. IMA Vol. Math. Appl. 42. Springer, New York, 1992.

[2] Bishop, R. L. And O'Neill, B.: Manifolds of negative curvature. Trans. Amer. Math. Soc. 145 (1969), 1-49.

[3] Greene, R. E. And Wu, H.: Function theory on manifolds which posses a pole. Lecture Notes in Mathematics 699. Springer, Berlin, 1979.

[4] Grigor'yan, A.: The heat equation on non compact Riemannian manifolds. Math. USSR Sb. 72 (1992) no.1, 47-77.

[5] Grigor'yan, A.: Analytic and geometric background of recurrence and non-explosion of the Brownian motion on Riemannian manifolds. Bull. Amer. Math. Soc. 36 (1999), no. 2, 135-249.

[6] Grigor'yan, A.: Heat kernels on weighted manifolds and applications. In The ubiquitous heat kernel, 93-191. Contemp. Math. 398. Amer. Math. Soc., Providence, RI, 2006.

[7] Grigor'yan, A. and Saloff-Coste, L.: Stability results for Harnack inequalities. Ann. Inst. Fourier 55 (2005) no. 3, 825-890.

[8] Hebish, W. and Saloff-Coste, L.: On the relation between elliptic and parabolic Harnack inequalities. Ann. Inst. Fourier 51 (2001) no. 5, $1437-1481$.

[9] Ishige, K. And Murata, M.: Uniqueness of nonnegative solutions of the Cauchy problem for parabolic equations on manifolds or domains. Ann. Scuola Norm. Sup. Pisa Cl. Sci. 30 (2001), no. 1, 171-223.

[10] March, P.: Brownian motion and harmonic function on rotationally symmetric manifolds. Ann. Probab. 14 (1986), no. 3, 793-801.

[11] Méndez-Hernández, P. J. And Murata, M.: Semismall perturbations, semi-intrinsic ultracontractivity, and integral representations of nonnegative solutions for parabolic equations. J. Funct. Anal. 257 (2009), no. 6, 1799-1827.

[12] Murata, M.: Structure of positive solutions to $(-\Delta+V) u=0$ in $\mathbb{R}^{n}$. Duke Math. J. 53 (1986), no. 4, 869-943.

[13] Murata, M.: Positive harmonic functions on rotationary symmetric Riemannian manifolds. In Potential theory, 251-259. De Gruyter, Berlin, 1991.

[14] Murata, M.: Uniqueness and nonuniqueness of the positive Cauchy problem for the heat equation on Riemannian manifolds. Proc. Amer. Math. Soc. 123 (1995), no. 6, 1923-1932.

[15] Murata, M.: Non-uniqueness of the positive Cauchy problem for parabolic equations. J. Differential Equations 123 (1995), no. 2, 343-387. 
[16] Murata, M.: Semismall perturbations in the Martin theory for elliptic equations. Israel J. Math. 102 (1997), 29-60.

[17] Murata, M.: Martin boundaries of elliptic skew products, semismall perturbations, and fundamental solutions of parabolic equations. J. Funct. Anal. 194 (2002), no. 1, 53-141.

[18] Murata, M.: Heat escape. Math. Ann. 327 (2003), no. 2, 203-226.

[19] Pinchover, Y.: Maximum and anti-maximum principles and eigenfunctions estimates via perturbation theory of positive solutions of elliptic equations. Math. Ann. 314 (1999), no. 3, 555-590.

[20] Saloff-Coste, L.: A note on Poincaré, Sobolev, and Harnack inequalities. Internat. Math. Res. Notices (1992), no. 2, 27-38.

[21] Saloff-Coste, L.: Parabolic Harnack inequality for divergence-form second-order differential operators. Potential Anal. 4 (1995), no. 4, 429-467.

[22] Tomisaki, M.: Intrinsic ultracontractivity and small perturbation for one dimensional generalized diffusion operators. J. Funct. Anal. 251 (2007), no. $1,289-324$.

Recibido: 26 de noviembre de 2009

Minoru Murata

Department of Mathematics Tokyo Institute of Technology Oh-okayama, Meguro-ku, Tokyo, 152-8551 Japan minoru3@math.titech.ac.jp 